\title{
ATLAS Forward Proton Detector
}

\section{Chiara Grieco*.}

Institut de Física d'Altes Energies (IFAE) Barcelona

Edifici CN UAB Campus, 08193 Bellaterra (Barcelona), Spain

E-mail: cgrieco@ifae.es

\begin{abstract}
The purpose of the ATLAS Forward Proton (AFP) detector is to measure protons scattered at very small angles. AFP aims to study diffractive events, exclusive production and photon induced interactions. The first arm of AFP with 3D pixel detectors was installed in early 2016 and data to study the tracker performance were recorded later that year during special low luminosity runs. The full two-arm setup (on both sides of the interaction point) was installed during the 2016/2017 LHC winter shutdown. In 2017, AFP participated in the ATLAS high-luminosity data taking on a daily basis. In addition, several special runs with reduced luminosity were taken. This paper presents the AFP detector and its performance during 2016 and 2017.
\end{abstract}

Sixth Annual Conference on Large Hadron Collider Physics (LHCP2018)

4-9 June 2018

Bologna, Italy

${ }^{*}$ Speaker. 


\section{Physics Motivation}

The ATLAS Forward Proton [1] consists of a Silicon Tracker (SiT) and Time-of-Flight (ToF) system housed in Roman Pot stations for the measurement of protons scattered at small angles. Such protons are predominantly produced in diffractive events when a colourless object is exchanged (for example a photon or a Pomeron). While diffractive scattering will be studied with AFP, the ultimate goal of the physics program is to focus on central exclusive events. For example, a precise measurement of the exclusive di-jet cross section will set constraints for other processes including the exclusive production of the Higgs boson. AFP can also be used as an effective tool for beyond Standard Model studies, or searching for anomalous quartic gauge couplings.

\section{Detector System}

AFP consists of four Roman Pot stations, two NEAR at $\sim 205 \mathrm{~m}$ and two FAR at $\sim 217 \mathrm{~m}$ away from the ATLAS IP. Each station includes part of the AFP detector system in a secondary vacuum environment (Roman Pot), allowing it to approach the LHC proton beam to $\sim 2 \mathrm{~mm}$. NEAR stations contain four layers of SiT detectors, while FAR stations contain four SiT modules and also a ToF system. Figure 1 shows a schematic of a Roman Pot station with the SiT and ToF system.

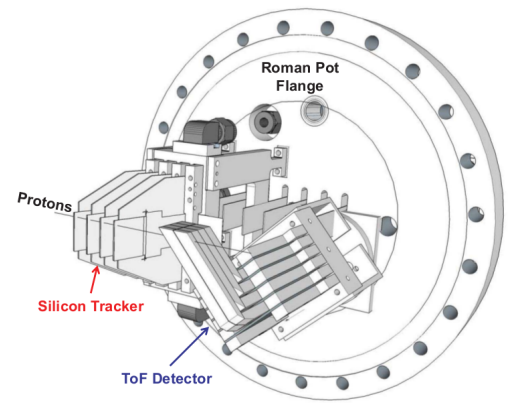

Figure 1: Sketch of Tracker and Time-of-Flight detectors mounted on a Roman Pot flange.

\subsection{Silicon Tracker $(\mathrm{SiT})$}

The goal of the AFP SiT is to precisely determine the path of the diffractive protons. This information, combined with the LHC optics allows to obtain the momentum transfer and the proton energy loss. The AFP tracker uses the 3D silicon pixel sensor technology, from CNM , which is already used in the ATLAS IBL [2] detector. The AFP 3D sensors differ from the IBL ones in that they present a slim edge of $\sim 180 \mu \mathrm{m}$ in the side closest to the LHC beam (IBL sensors have an inactive edge of $\sim 1 \mathrm{~mm}$ in this direction). The sensor consists of $336 \times 80$ pixels of $50 \times 250 \mu \mathrm{m}^{2}$. They are read out by the ATLAS FEI4 ASIC [3]. This ASIC provides a tunable threshold, charge measurements via the Time-over-Threshold (ToT) information, and a trigger signal. The AFP modules were shown to provide radiation hardness to non-uniform fluence of up to $3 \cdot 10^{15} \mathrm{n}_{\mathrm{eq}} / \mathrm{cm}^{2}$ 
${ }^{1}$ and excellent position resolution of $6 \mu \mathrm{m}(70 \mu \mathrm{m})$ in the short (long) pixel direction when tilted by $14^{\circ}$ with respect to the incident protons [4].

\subsection{Time-of-Flight (ToF)}

In order to precisely obtain the time of arrival of the particle a Time-of-Flight (ToF) system is needed. The ToF system is a matrix of $4 \times 4$ quartz bars mounted at the Cherenkov angle with respect to the diffractive proton direction. An ultrafast photomultiplier (MCP-PMT) converts the light from the bars into electric pulses which are further amplified and read out. The expected resolution from test beams and early analysis is $20-30$ ps per train [4]. While primarily meant for pile-up removal, the ToF system can also provide the trigger decision for low and high luminosity runs.

\section{AFP Performance in 2016}

The first AFP performance studies were already presented in [5]. The data sample considered was collected in October 2016 using a special LHC beam configuration such that the average number of interactions per beam crossing was limited to $\mu \sim 0.3$ with the standard LHC optics $\left(\beta^{*}=0.4\right.$ $\mathrm{m})$. At that time, AFP consisted of one arm with two stations with four 3D pixel tracking detectors each. A data sample with tagged protons was selected using a trigger that required events with at least four AFP hits (two or more per station) and at least one jet in the ATLAS calorimeter with $p_{T}>10 \mathrm{GeV}$. Events were required to have a single reconstructed primary vertex consistent with the beam spot and to contain at least two associated tracks. Events with multiple reconstructed vertices were rejected. Events were required to have at least five AFP tracker planes with one cluster each and at least one jet in the calorimeter with $p_{T}>20 \mathrm{GeV}$ and $|\eta|<3$.0. Also events were rejected if they contained any identified pile-up jet. A separate sample was collected using a minimum bias (MB) trigger, looking for the presence of 2 hits in the ATLAS Minimum Bias Trigger Scintillator (MBTS) [6], and a jet of $p_{T}>20 \mathrm{GeV}$ in the final state. This sample is dominated by non-diffractive events. The fraction of the proton momentum participating in the hard interaction is estimated using calorimeter information in the ATLAS detector using the expression $\xi_{\text {cal }}=\frac{1}{\sqrt{s}} \sum_{i} p_{T}^{i} e^{-\left(\eta_{i}\right)}$ where the sum runs over all the calorimeter clusters with $p_{T}$ above $200 \mathrm{MeV}$. The measured uncorrected $\xi_{\text {cal }}$ distributions at the detector level are shown in Figure 2. The AFPtriggered data sample exhibits a double peak structure dominated by diffractive processes at low $\xi_{c a l}$ and non-diffractive processes at large $\xi_{c a l}$. The presence of the peak at low $\xi_{\text {cal }}$ indicates that there is a correlation between AFP triggers and diffractive events in the central detectors.

\section{SiT Performance in 2017}

In the shutdown of 2016-2017 the AFP detector was completed by installing the second AFP arm and the ToF system in the FAR stations. However the ToF efficiency was extremely low due mostly to the quick gain degradation of the MCP-PMT caused by radiation. In 2017, data at nominal luminosity were collected during special runs. For these, data analysis is ongoing. One

\footnotetext{
${ }^{1}$ Where $\mathrm{n}_{\mathrm{eq}}$ indicates the equivalent for $1 \mathrm{MeV}$ neutron.
} 


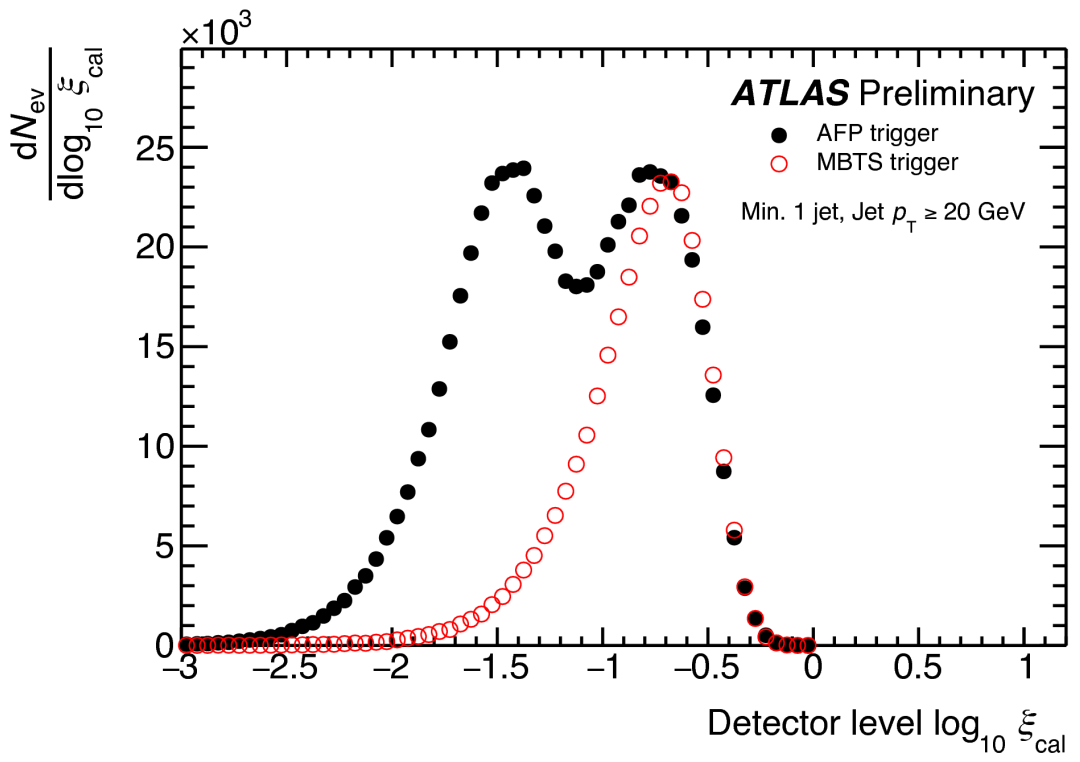

Figure 2: Measured $\xi_{c a l}$ distribution in AFP and MB selected data samples on linear scale, for events with at least one jet with $p_{T}>20 \mathrm{GeV}$ and $|\eta|<3.0$. The MB data is normalized to the AFP data in the large $\xi_{c a l}$ region [5].

of the critical aspects of the AFP detector is the performance of AFP tracker. This needs constant monitoring due to the detrimental effect of radiation on the tracker modules. Thus, the hit efficiency of the tracker planes as a function of Bias Voltage (HV) is studied periodically.

\subsection{Tracker Performance in 2017}

Tracks are reconstructed using three planes, and the extrapolated position (interpolated) from the track is compared to the hit positions in the fourth plane, called Device Under Test (DUT). The track reconstruction requires a single cluster per plane, consisting of no more that two pixels. Figure 3 (up) shows the extrapolated position (X,Y) in Plane 0, Station A NEAR at 60V. Three different occupancy regions with different track multiplicities are defined in order to study efficiency as a function of HV and fluence. The high occupancy region is the region where the occupancy is between $70 \%$ and $100 \%$, medium occupancy is the region from $30 \%$ to $70 \%$, and low occupancy region is where the occupancy is less than $30 \%$. The efficiency is evaluated matching the extrapolated position from the reconstructed track with the hit position in the DUT plane in a window of one pixel $(50 \mu \mathrm{m}$ on $\mathrm{X}$ and $250 \mu \mathrm{m}$ on $\mathrm{Y})$, and normalizing by the total number of tracks. In Figure 3 (bottom) the efficiency for the three different occupancy regions and as a function of the bias voltage is shown. Full efficiency is recovered for all the regions for a bias voltage larger than $30 \mathrm{~V}$.

\section{Conclusions}

AFP was installed in 2016 with only one arm with two tracker stations. Data were taken during special low pile-up runs. The AFP detector is capable to identify diffractive protons. In 

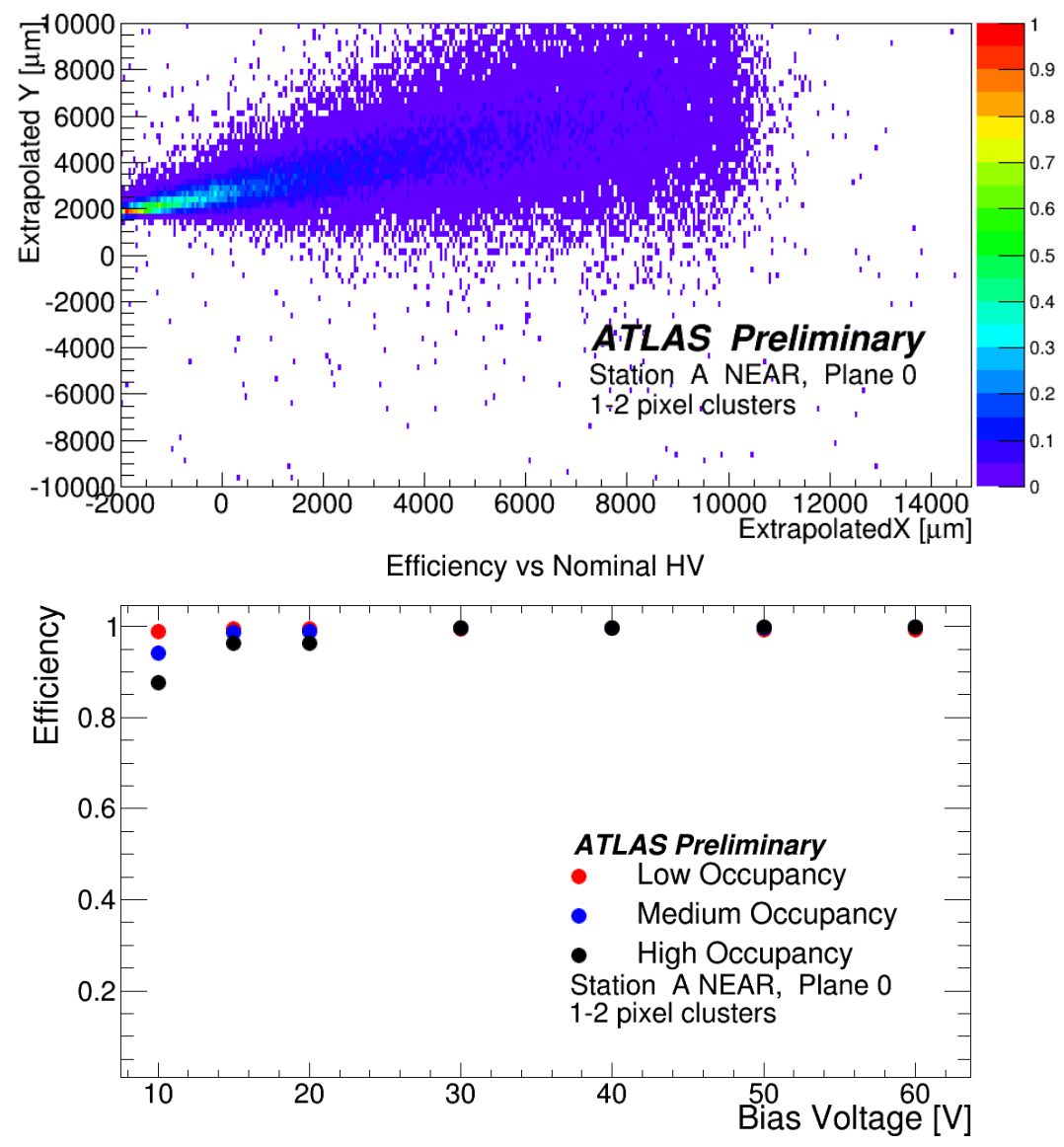

Figure 3: Up: Extrapolated position in Station A NEAR, Plane 0 using the track reconstructed by planes 1 , 2 and 3. Down: Efficiency for different HV and different occupancy regions

2017 the full system was installed: two arms with two station each. During standard and special runs $32 \mathrm{fb}^{-1}$ of data were recorded. The AFP tracking performance is being monitored to ensure excellent efficiency.

\section{References}

[1] The ATLAS Collaboration, The ATLAS Forward Proton Detector, Tech. Rep. CERN-LHCC-2015-009. ATLAS-TDR-024, May, 2015.

[2] The ATLAS Collaboration, ATLAS Insertable B-Layer, Tech. Rep. CERN-LHCC-2010-013. ATLAS-TDR-19, September, 2010.

[3] M. Garcia-Sciveres et al., The FE-I4 readout integrated circuit, Nucl. Instr. Meth. 1A 636 (2011).

[4] J. Lange et al., Beam tests of an integrated prototype of the ATLAS Forward Proton detector, JINST 11 (2016) P09005.

[5] The ATLAS Collaboration, Proton tagging with the one arm AFP detector, ATL-PHYS-PUB-2017-012, July, 2017.

[6] A. Sidoti, Minimum Bias Trigger Scintillators in ATLAS Run II, JINST 9.10 (2014) C10020. 